\title{
Dynamic Path Reconfiguration among Hybrid FSO/RF Nodes
}

\author{
Swapna Gurumani ${ }^{*}$, Hassan Moradi ${ }^{*}$, Hazem H. Refai ${ }^{*}$, Peter G. LoPresti ${ }^{* *}$, Mohammed Atiquzzaman ${ }^{* * *}$ \\ "Electrical and Computer Engineering, University of Oklahoma, Tulsa, OK. \\ (Tel: 918 660-3243; e-mails: swapnagg@ou.edu,hmoradi@ou.edu,hazem@ou.edu \\ ${ }^{* *}$ Electrical Engineering Department, University of Tulsa, Tulsa, OK. \\ (Tel: 918 631-3274, e-mail: pgl@ohm.ee.utulsa.edu) \\ ${ }^{* * *}$ Computer Science, University of Oklahoma, Norman, OK. \\ (Tel: 405 325-8077; email: atiq@ou.edu)
}

\begin{abstract}
To increase the availability of Free Space Network (FSO) networks, the optical link is provided with a redundant backup network, such as RF, to form a hybrid network. The immediate switching from FSO to RF in hybrid networks leads to suboptimal utilization of FSO network. In this work, a network of hybrid nodes, where each node is equipped with multiple transceivers and has multiple associations with other nodes of the network is proposed. Alternate FSO paths are established and prioritized through these multiple associations via intermediate nodes. On the loss of an active FSO link, path reconfiguration takes place, and the FSO path next in priority is utilized. Thus, communication can be maintained in the optical domain for a longer period, and efficiency is increased. A hardware testbed with four hybrid nodes having multiple IR transceivers to establish optical links was constructed to validate proof of concept. Dynamic path reconfiguration in the optical domain was demonstrated in the testbed by blocking different transceivers of the nodes. We measure network performance in terms of end-to-end delay along alternative FSO paths. Results show how the proposed path reconfiguration algorithm can provide performance improvement in hybrid networks.
\end{abstract}

\section{INTRODUCTION}

The possibility of integrating FSO link with an RF link as a redundant backup link has recently been explored in communication networks. Combining these two technologies to form a hybrid network is logical because of their complimentary nature with regard to weather sensitivity. The result is a network that provides more reliable communication with increased quality of service $[1,2,3]$.

A number of commercially available hybrid networks [5] deploy FSO as a primary high bandwidth network with parallel RF as secondary or backup to achieve a highly available network. An outage of the FSO link immediately triggers the activation of the secondary RF link to ensure seamless connectivity. Since RF routes has less bandwidth compared to FSO routes, more than one RF routes should be used in order to maintain QoS between source and destination. However, in a hybrid network with multiple nodes, such an immediate substitution with RF results in suboptimal utilization of the FSO network. To maximize the advantages, e.g. high data rate, of FSO, the network must remain in the FSO domain as long as possible. The aim of this research is to achieve this advantage using the concept of multi-connected nodes.

Several studies targeting disparate issues in hybrid FSO/RF networks are available in the literature. Performance and throughput evaluation under various weather conditions was performed using simulation or testbeds $[5,6]$. Some weather conditions, such as cloud, were statistically characterized, and different configurations for the ground and air nodes were chosen [5]. Algorithms have been proposed [7], where a framework of modeling failures and path reconfiguration for traffic in hybrid RF/FSO networks is detailed. Given known traffic and physical network conditions, topology control combines algorithms for topology reconfiguration with pointing acquisition and tracking (PAT) techniques to dynamically reform the network, thereby improving QoS and throughput [8]. PAT considerations have been widely studied in $[9,10]$.

The network performance in the form of end-to-end delay for different obscuration scenarios and packet drop rate is extensively calculated in [11]. But, our research emphasizes on such delay in implementation aspects and is driven by the need to provide reliable wireless broadband connectivity with low end-to-end delay. The contributions of this study are as follows:

- Proposal and implementation of a hybrid FSO/RF network that will establish a path and perform path reconfiguration in the event of a loss of an active optical link.

- Construction of a testbed with four (4) nodes that uses inexpensive and easily available components.

- Experimental verification of path reconfiguration algorithms along multiple paths between a given transmitter-receiver pair via intermediate nodes that uses the multi-connected nature of the nodes.

The main objective of the current work was to develop a hybrid network using FSO and RF that provides maximum capacity and availability while utilizing the highest FSO network potential. To achieve this, a hybrid network prototype using nodes with multiple optical transceivers is proposed. A hardware testbed consisting of several nodes--each equipped with multiple transceivers--is described. In a process called Topology Discovery, multiple optional paths are formulated between two given pairs of nodes prior to actual data transmission. Quick path re-routing through available alternate paths occurs, thus maintaining almost uninterrupted connectivity in the event of FSO link failure. Connectivity in the optical realm is retained as long as possible, with a switch to RF only occurring as the final option. A robust, flexible, reliable and high bandwidth hybrid network is achieved. 
The paper is organized as follows: Section II gives a brief description of routing and reconfiguration in hybrid networks. Section III discusses our network model and configuration definition, along with failure modeling. Section IV presents our proposed algorithms for integrated RF topology control and routing. Section $\mathrm{V}$ describes implementation results, and Section VI provides a conclusion for the paper.

\section{ROUTING AND RECONFIGURATION}

The main challenge for mobile $\mathrm{FSO} / \mathrm{RF}$ networks that consist of hybrid directional RF and narrow beam FSO links is the dynamic, autonomous reconfiguration of both hardware and software so that communication availability and capacity for tactical operations is maximized. We refer to this inclusive process as topology control [9]. (It should be noted that the current work does not involve optical communication terminal design, as the various aspects that must be considered [10] are challenging.) Reconfiguration occurs physically, by means of PAT, and logically, using autonomous reconfiguration algorithms and heuristics; it occurs in response to monitored degradation of one or more links in the network. Maximum availability and capacity can be provided most effectively by having diverse operational options, which utilize an integrated hardware and software approach.

Rerouting on FSO topology for the remaining failed traffic is possible, although a certain delay is incurred. As a result, a routing scheme is proposed to maximize the throughput on the FSO topology and maximize the minimum criticalityweighted backup provided to the traffic on the RF topology [7]. A routing algorithm is required for calculating an RF topology and backup routing for a given FSO topology and routing.

\section{NETWORK MODEL}

We consider a hybrid RF/FSO backbone network where each node is capable of routing [12]. In this section, we discuss the actual network topology establishment, path reconfiguration, and data rerouting which will be used to illustrate our proposed algorithms for integrated RF topology control and routing.

\section{A. Assumptions and Requisites}

The following assumptions and stipulations are made in this research; however, they don't contradict the real environment and don't affect the authenticity of the analysis.

1. Free Space Optics is used for data only, and RF is used primarily for Control Signals and for data transfer whenever FSO is unavailable.

2. The RF channel is $100 \%$ available.

3. Simplex communication among nodes.

4. The transmitter broadcasts data continuously.

5. Each node is equipped with multiple transceivers for interference free communication with every other node.

6. No Acquisition and Pointing Algorithm is performed.

7. The FSO Link becomes unavailable due to one or more of the following reasons: Poor atmospheric conditions, device failure, or loss of line of sight due to misalignment.

The RF channel, which is assumed to be always available, is intended to be a backup network for improved reliability and QoS of the FSO network. The RF channel is used primarily for control signals; it is used for data only in the event of a complete outage of the FSO network. Due to the simplex nature of the testbed, only one pair of transmitter and receiver communicates serial data in a continuous loop. The other nodes act as intermediate nodes and reroute data from the transmitter to the receiver. The FSO network is configured to transmit at three times the rate of RF. All the nodes are static, and loss of line-of-sight will cause loss of link. The most important feature of the testbed is that each node has multiple transceivers for having multiple associations with other nodes.

\section{B. Topology Discovery}

Network establishment process starts with each node attempting to ping its neighboring nodes through its transceivers. Depending on the response, each node becomes aware of its neighbors. Prior to data transmission, the transmitter signals its neighbors to obtain information to aid in the formation of a routing table. Hence, the transmitter is aware of the entire network topology. This process, termed topology discovery, is illustrated in Figure 1 in the form of a signaling diagram. The routing table complied by the Primary Transmitter at the end of the topology discovery process is shown in Table 1. The topology discovery process is an important precursor to actual data communication because it creates multi-connected nodes, i.e. each node has multiple associations established along multiple interfaces. Thus, multiple paths exist between a given source-destination pair which are determined and listed beforehand in order of priority. The path with either the least delay or least number of hops is given highest priority, whereas the path with either the maximum delay or maximum number of hops has the lowest priority. Routing algorithms can then be implemented to reroute data through these alternate paths in the event of the loss of an active link.

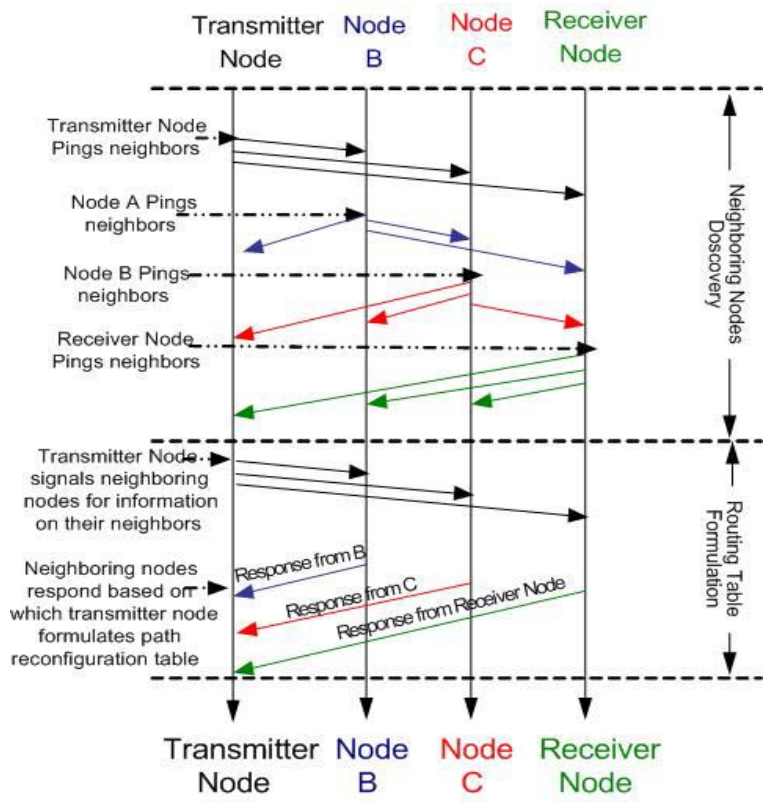

Figure 1: Topology Discovery signaling diagram.

Table 1: Routing Table formulated by Primary Node

\begin{tabular}{|c|c|c|c|c|c|}
\hline Source & Destination & Path & $\begin{array}{c}\text { Number of Nodes } \\
\text { Involved }\end{array}$ & $\begin{array}{c}\text { Hop } \\
\text { Distance }\end{array}$ & Priority \\
\hline Transmitter Node & Node B & 1 & 2 & 1 & 1 \\
\hline Transmitter Node & Node B & 2 & 3 & 2 & 2 \\
\hline Transmitter Node & Node B & 5 & 4 & 3 & 3 \\
\hline Transmitter Node & Node C & 1 & 2 & 1 & 1 \\
\hline Transmitter Node & Node C & 2 & 3 & 2 & 2 \\
\hline Transmitter Node & Node C & 5 & 4 & 3 & 3 \\
\hline
\end{tabular}


As an example, Table 1 illustrates some of the alternate paths between the transmitter and receiver nodes that are computed with priority assigned to each path in the current 4nodes model.

\section{PATH RECONFIGURATION}

The topology discovery process described in the previous section is crucial for the path reconfiguration process. This is due to the fact that all possible paths between a given pair of nodes are computed and tabulated in the order of priority as determined during the topology discovery process. A path reconfiguration process occurs as a result of loss of an active link caused by one or more of the aforementioned reasons, e.g. loss of line-of-sight, atmospheric conditions, or device failure. During such an event, the path next in the list is chosen, and, data are re-routed. Since all routes are formulated in advance, path reconfiguration occurs with the least possible delay. This section details the path reconfiguration algorithm.

The transmitter broadcasts data through FSO using the direct path with the highest priority. In case of a link loss during data transfer, the receiver immediately alerts the transmitter through the RF control channel. The transmitter continues data transfer through the path next in the priority list. This is how an alternate path is chosen. Figure 2 shows the path reconfiguration algorithm in the form of flow chart. It should be noted that the network attempts to transmit data using FSO as long as possible. However, this is not possible if none of the FSO paths formulated in the topology discovery process are available. In the absence of a FSO link, data transmission occurs through the direct RF path between the transmitter and receiver. Clearly, the consequence of switching to the RF network occurs only after all FSO paths are attempted and unavailable.

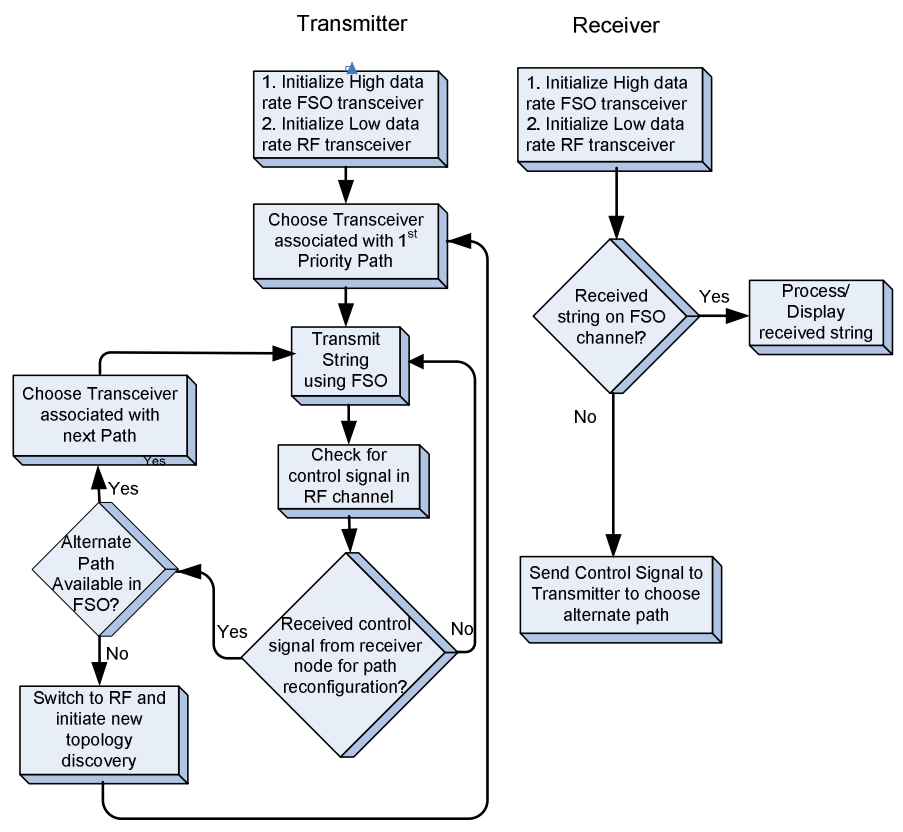

Figure 2: Path reconfiguration algorithm.

It is important to note that each time the receiver stops receiving data, it automatically assumes the FSO link is broken and sends a control signal to the transmitter in an attempt to establish an alternate path. The transmitter immediately employs the next path found during topology discovery, thereby avoiding wasting time for attempting to resend data through the current path. If the transmitter is unable to communicate with the receiver after evaluating all possible paths, only then the backup RF network is utilized and a new topology discovery process initiated.

\section{IMPLEMENTATION ASPECTS AND RESULTS}

In this section, we describe the results obtained from the implementation of our proposed path reconfiguration using the laboratory testbed [12]. We used a dragon12 board equipped with Motorola $68 \mathrm{HC} 12$ microcontroller for each node, and two sets of serial transmission and reception ports: one reserved for FSO and the other reserved for RF.

The optical link broadcasts serial data continuously through a serial transmitter using the IRDA module. Loss of the link is caused by manually blocking the IRDA transmitter. When data reception is blocked, the receiver immediately interprets the event as a loss of FSO link and notifies the transmitter through the serial transmitter associated with its RF link. The transmitter then sends a control signal to the address decoder triggering it to choose another transceiver, thus linking to another node. The FSO serial port is initialized to both transmit and receive at $9.6 \mathrm{kbps}$, and the RF serial port is initialized to $2.4 \mathrm{kbps}$.

\section{A. Measurement Procedure}

Fig. 3 shows our testbed consisting of a network of four transceiver nodes. Each node can communicate with all other nodes through both FSO and RF. To eliminate interference with other transceivers, the field of view of the transceiver is kept narrow. Different transceivers were manually blocked with black tape to force the system to establish all alternate routes between the transmitter and receiver. This is illustrated in Figure 3. Thus, the path reconfiguration algorithm was demonstrated using the testbed and verified through experimentation. The start of transmission at the transmitter is marked as the transmitted time. Similarly, the start of reception of the transmitted string at the receiver is marked as the received time. To mark these transmission and reception instances, a Tektronix Mixed Signal Oscilloscope (MSO) is used. By marking the transmission and reception instances, the end-to-end delay for different paths and the time for path reconfiguration due to loss of an active link are obtained.

\section{B. Measured End-to-End Delay}

The measured end-to-end delay in milliseconds for different paths is tabulated in Table 2 [12]. The source and the destination for all paths are the transmitter node and the receiver node, respectively. Column 3 of Table 2 indicates the Intermediate nodes participating, if any, and their sequence along each path. The end-to-end delay in the present context, is measured between the output pin in the transmitter node and the input pin of the receiver node for all paths.

Table 2: Measured end-to-end delay (in ms) for different paths

\begin{tabular}{|c|c|c|c|c|c|}
\hline Path & Source & $\begin{array}{c}\text { Intermediate } \\
\text { Node(s) }\end{array}$ & Destination & $\begin{array}{c}\text { No. of } \\
\text { hops }\end{array}$ & $\begin{array}{c}\text { Delay } \\
(\mathrm{ms})\end{array}$ \\
\hline 1 & $\mathrm{~T}$ & $\mathrm{X}$ & $\mathrm{R}$ & 1 & 0.1 \\
\hline 2 & $\mathrm{~T}$ & 1 & $\mathrm{R}$ & 2 & 2.2 \\
\hline 3 & $\mathrm{~T}$ & 2 & $\mathrm{R}$ & 2 & 2.2 \\
\hline 4 & $\mathrm{~T}$ & 1,2 & $\mathrm{R}$ & 3 & 4.3 \\
\hline 5 & $\mathrm{~T}$ & 2,1 & $\mathrm{R}$ & 3 & 4.3 \\
\hline
\end{tabular}




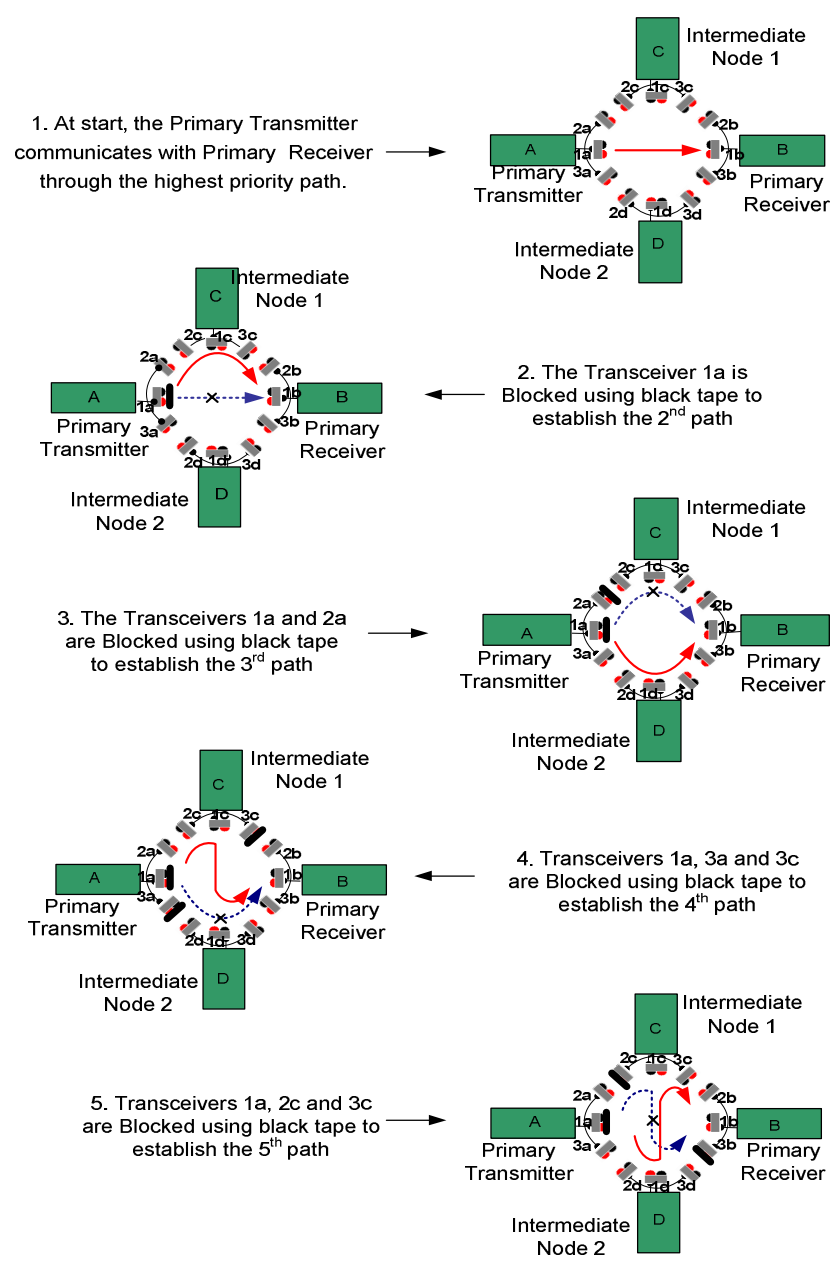

Figure 3: Illustration of establishment of alternate routes for different failure scenarios.

An explanation of the measured end-to-end delay is given below:

1. Delay along path 1: The transmitted optical data is received by the IR transceiver module in the receiver node (MCP2120) and demodulated and decoded at $9.6 \mathrm{Kbps}$. This is shown in Figure 4 where ' $a$ ' and ' $b$ ' represent the two end points for measurement. Therefore, each bit appears at the rate of almost 0.1 milliseconds at the receiver node, which is the measured delay for the direct path.

Let $T_{a b}$ be the time taken for data to reach from the output pin of the transmitter to the input pin of the receiver. Therefore delay for path 1:

$$
T_{a b}=(1 / 9600)=0.1 \mathrm{~ms}
$$

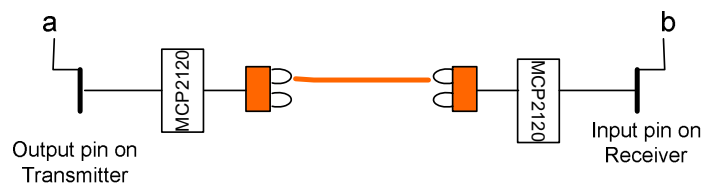

Figure 4: Delay for path 1 measured between points ' $a$ ' and 'b'.

2. Delay along path $2 \&$ path 3 : In path 2 and path 3 , the data transmitted by the transmitter node is routed to the receiver node through an intermediate node. This is shown in Figure 5. Delay is measured between ' $a$ ' and ' $d$ '.
$T_{a b}=T_{c d}=0.1 \mathrm{~ms}$, from (1) and $T_{b c}$ is the time taken for the receiver microcontroller to read data from the input pin, display the data on the LCD screen, execute software instructions, and transmit data to the output pin (data is read once for every eight received bits and transmitted at the same rate)

$$
T_{b c}=0.8+0.4+0.8=2 \mathrm{msec}
$$

Delay analog path 2 and path 3 is

$$
T_{a d}=T_{a b}+T_{b c}+T_{c d}=2.2 \mathrm{msec}
$$

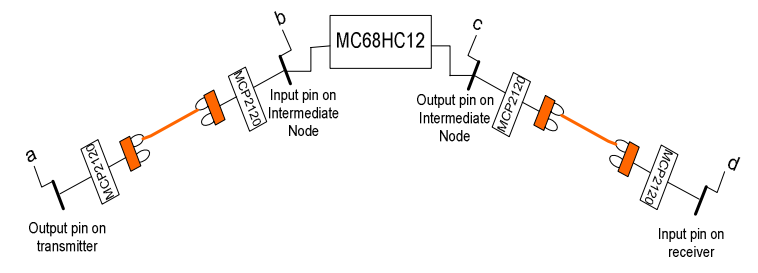

Figure 5: Delay for path $2 \& 3$ measured between points 'a' and 'd'

3. Delay along path $4 \&$ path 5 : In path 4 and path 5 , the data transmitted by the transmitter node is routed to the receiver node through two intermediate nodes. This is shown in Figure 6. Delay is measured between ' $\mathrm{a}$ ' and ' $\mathrm{f}$ '.

$T_{a b}=T_{c d}=T_{e f}=0.1 \mathrm{msec}$, from (1) and $T_{b c}=T_{d e}=2$ $\mathrm{msec}$, from (2). So, the delay along path 4 and path 5 is

$$
T_{a f}=T_{a b}+T_{b c}+T_{c d}+T_{d e}+T_{e f}
$$

The end-to-end-delay along different paths increases with an increase in the number of intermediate nodes.

\section{Measured path reconfiguration time}

To determine the path reconfiguration time, active links were blocked to establish new paths, and the time taken to establish the new path was determined. Twenty trials were made for this purpose, and the result is summarized in Table 3. An explanation for the results obtained is given below.

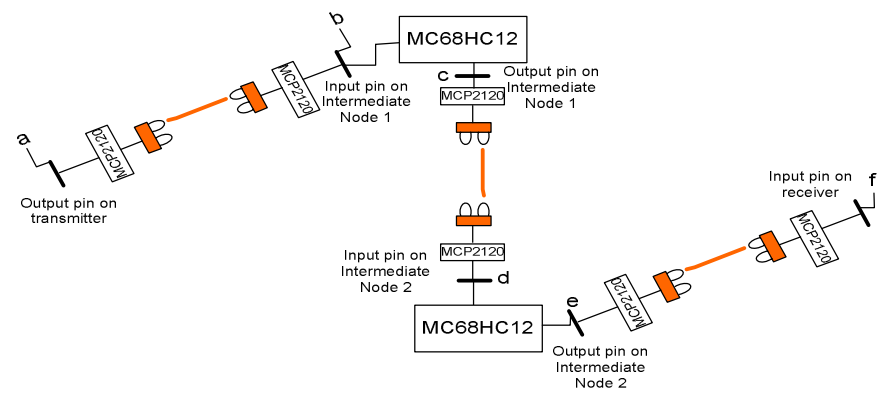

Figure 6: Delay for path $4 \& 5$ measured between points ' $\mathrm{a}$ ' and ' $\mathrm{f}$ '

Table 3: Time taken for path reconfiguration in 20 trials

\begin{tabular}{|l|c|}
\hline Minimum time taken for path reconfiguration & $17 \mathrm{msecs}$ \\
\hline Maximum time taken for path reconfiguration & $80 \mathrm{msecs}$ \\
\hline Average time taken for path reconfiguration & $40.4 \mathrm{msecs}$ \\
\hline Most frequent time taken for path reconfiguration & $40 \mathrm{msecs}$ \\
\hline
\end{tabular}

The path reconfiguration time consists of three parts:

- Time taken for receiver to discover an interruption in service $\left(T_{l}\right)$. This parameter represents the time that receiver waits for the packet before generating interrupt. 
- Time taken for the receiver node to transmit the control signal to the transmitter node and the transmitter node to read this information $\left(T_{2}\right) . T_{2}$ is a constant. Since this occurs at the rate of $2.4 \mathrm{Kbps}$ and data is sent out and received 8 bits at a time,

$$
T_{2}=2 * 8 *(1 / 2400)=6.6 \mathrm{~ms}
$$

- Time taken for the data to reach the receiver through the new path $\left(T_{3}\right)$. Depending on the path chosen, this value is either $0.1 \mathrm{~ms}, 2.2 \mathrm{~ms}$ or $4.3 \mathrm{~ms}$ (Eqn 1, 3, 4). Therefore, total time taken for path reconfiguration is:

$$
T_{p r}=T_{1}+T_{2}+T_{3}
$$

The performance is tested in terms of average delays while dropped packets consideration is not in the scope of this paper.

\section{Limit of path reconfiguration time}

If data were received at the $61^{\text {st }}$ millisecond and interruption occurs thereafter, then the instance at which the received flag is polled at the $70^{\text {th }}$ millisecond will remain set. It must wait an additional 30 milliseconds to be cleared, and the interruption in service will be discovered once it is polled again. Thus, the maximum value for $T_{1}$ is 79 milliseconds. $T_{2}$ is always 6.6 milliseconds. Maximum value for $T_{3}=4.3$ milliseconds. Therefore, the maximum value for path reconfiguration time is

$$
79+6.6+4.3=89.9 \mathrm{~ms}
$$

If an active link was broken at $59^{\text {th }}$ millisecond, then the "received" flag is cleared at the $60^{\text {th }}$ millisecond. Thereafter, when the received flag is polled at the $70^{\text {th }}$ millisecond it will be remain cleared. Thus the minimum value for $T_{1}$ is $10 \mathrm{~ms} . T_{2}$ is always $6.6 \mathrm{~ms}$. Minimum value for $T_{3}=0.1 \mathrm{~ms}$. Therefore, the minimum value for path reconfiguration time is

$$
10+6.6+0.1=16.7 \mathrm{~ms}
$$

Thus, the path reconfiguration time can range from $16.7 \mathrm{~ms}$ to $89.9 \mathrm{~ms}$. If the polling frequency is increased, the time for path reconfiguration reduces. However, this event caused an unstable condition in the testbed. Hence, an optimum value of $70 \mathrm{~ms}$ was chosen to create a quick, but stable response.

Since reconfiguration time period is always larger than transmission time period in simulation, a retransmission mechanism may be used to recover the data which are lost during recovering time period. The transmitted message can be also duplicated on the RF channel allowing data recovery during the failure of the optical wireless channel.

\section{CONCLUSIONS}

This work illustrates the enterprise of formulating rerouting of data between primary and secondary optical paths between a transmitter and receiver via intermediate nodes due to multiple associations. Loss of a primary optical path triggers the transmitter to attempt to switch to an alternative secondary optical path before finally switching to the RF path, ensuring maximum optical connectivity period. This concept was implemented and experimentally demonstrated using a hardware testbed. A number of combinations of transceivers were blocked to cause loss of optical link, and subsequently, the network performance was studied. The delay along different optical paths and time taken for path reconfiguration were experimentally determined and presented.

This work verified the notion of reconfigurable topology resulting from alternative FSO paths between a given pair of communicating nodes provides increased availability of the high throughput optical network. Network performance in the form of end-to-end delay was measured for different routing paths. The results conclusively show acceptable improvement with topology control and proposed reconfiguration algorithm. Experimental verification of path reconfiguration algorithms using the multi-connected nature of the nodes was done and we believe that the results outperform the results derived in [11]. This can be figured out by comparing the values of average end to end delays. Furthermore, this paper issues the results of an algorithm experimentally (not by simulation) achieved.

\section{ACKNOWLEDGMENT}

This work is funded by NSF grant number NSF-ECCS 0725801 .

\section{REFERENCES}

[1] Kashyap, A. Shayman, M., Routing and traffic engineering in hybrid RF/FSO networks, 2005 IEEE International Conference on Communications, Volume 5, pp. 3427- 3433, May 2005.

[2] Vangala, S., Pishro-Nik, H., A Highly Reliable FSO/RF Communication System Using Efficient Codes, Proceedings of the IEEE GLOBECOM '07, pp. 2232-2236, Nov. 2007.

[3] Gauger, C.M., Kuhn, P.J., Breusegem, E.V., Pickavet, M., Demeester, P., Hybrid optical network architectures: bringing packets and circuits together, IEEE Communications Magazine, Volume 44, Issue 8, pp. 36 - 42, Aug. 2006.

[4] Free space optics Vs RF, Available: http://www.freespaceoptic.com/fsovsrf.htm (visited Dec 2007).

[5] Wu, H., Hamzeh, B. and Kavehrad, M., Availability of airborne hybrid FSO/RF links, Proceedings of the SPIE, Volume 5819, pp. 89-100, June 2005.

[6] Derenick, J., Thorne, C., Spletzer, J., Hybrid Free-Space Optics/ Radio Frequency (FSO/RF) Networks for mobile robot teams, in Multi-Robot Systems: From Swarms to Intelligent Automata, Alan C. Schultz and Lynne E. Parker (eds.), Springer, March 2005.

[7] Kashyap, A. Rawat, A., Shayman, M., Integrated Backup Topology Control and Routing of Obscured Traffic in Hybrid RF/FSO Networks, Proceedings of the IEEE GLOBECOM'06, pp. 1-6, Nov. 2006.

[8] Zhuang, J., Casey, M.J., Milner, S.D., Gabriel, S.A., Baecher, G.B., Multi-objective optimization techniques in topology control of free space optical networks, Proceedings of the IEEE MILCOM 2004, Vol. 1, pp. 430-435 Vol. 1, Nov. 2004.

[9] Ho, T., Trisno, S., Smolyaninov, I., Milner, S., Davis, C. Studies of Pointing, Acquisition, and Tracking of Agile Optical Wireless Transceivers for Free Space Optical Communication Networks" SPIE Conference, Remote Sensing 2003, Barcelona, Spain, Vol. 5237, pp. 147-158, 2003.

[10] Epple, B., Henniger, H., Discussion on Design Aspects for FreeSpace Optical Communication Terminals, IEEE Communications Magazine, pp. 62-69, October 2007.

[11] Llorca, J., Desai, A., Baskaran, E., Milner, S., Davis, C., Optimizing Performance of Hybrid FSO/RF Networks in Realistic Dynamic Scenarios, Proc. SPIE, Vol. 5892, pp. 589207-1-9, 2005.

[12] Guramani, S., M., Implementation of Dynamic Path Reconfiguration among Hybrid FSO/RF Nodes, Master of Science thesis, The University of Oklahoma, Tulsa, OK, 2007. 